\title{
Design of a Microstrip Balanced Amplifier Using the Wilkinson Power Divider
}

\author{
Amine Rachakh ${ }^{1}$, Larbi El Abdellaoui ${ }^{2}$, Ahmed Errkik $^{3}$, Abdelali Tajmouati ${ }^{4}$, Mohamed \\ Latrach $^{5}$ \\ \{rachakh.amine1@gmail.com¹, elabdellaoui20@hotmail.com², ahmed.errkik@uhp.ac.ma3 $\}$ \\ 1,2,3,4MEET Laboratory, FST of Settat, Hassan $1^{\text {st }}$ University, Morocco \\ ${ }^{5}$ Microwave Group, Ecole Supérieure d'Electronique de l'Ouest, Angers, France
}

\begin{abstract}
The broadband bandwidth of power amplifiers is one of the most important features that restrict its wide usage. This work presents a new structure of a broadband power amplifier suitable for ISM band. The proposed amplifier, which is realized with two identical single-stage amplifiers and two power dividers on low loss Epoxy (FR4) substrate with a substrate thickness of $1.6 \mathrm{~mm}$ and a relative permittivity equal to 4.4 . The proposed PA demonstrates a power gain of $16 \mathrm{~dB}$ at $2.45 \mathrm{GHz}$. The input and output matching impedances are better than $-15 \mathrm{~dB}$ throughout the band. the power amplifier delivers a maximum output power equal to $16 \mathrm{dBm}$ with a flat gain varied between $15-16 \mathrm{~dB}$.
\end{abstract}

Keywords: Power Amplifier (PA), Gallium Arsenide (GaAs), Field-effect transistor (FET), Microstrip technology, Advanced Design System (ADS).

\section{Introduction}

Broadband power amplifiers are critical circuit components for wireless communication systems, as well as cellular telephone applications. The broadband power amplifier should be designed to trade-off the power gain, output power, return loss and bandwidth. The broadband amplifier can be designed by utilizing the compensation network, but the mismatching will raise the VSWR [1]. When VSWR improve, the power gain and output power will deteriorate. The balanced power amplifier can equilibrium these performance parameters for its coupling circuit at the input and output ports.

Conventionally, balanced power amplifiers are made up by associating two identical amplifiers with branch line couplers at the input and output as illustrated in Figure 1. Based on the circuit configuration illustrated in Figure 1. Balanced power amplifiers have been realized with various types of couplers, such as broadside couplers [2-3], branch-line couplers [4-5], and Lange couplers [6-7].

Various technologies are utilized in designing PA for several applications in wireless communications. For microwave frequencies, the GaAs MESFET technology appears to be prominent. This is owing to it has superior electrical characteristics at high frequencies, which helps to transmit high output power with a minimum parasitic loss. Moreover, its inherent characteristic of low resistance and low drain-source offset voltage [8-10]. 
Following these studies, this paper presents a new design of a balanced power amplifier by using a Wilkinson power divider. To improve circuit performance, the microstrip lines technology is used. While in the design process, the advanced design system (ADS) software is utilized, and it can also give simulation result of amplifier final circuit such as power gain, reflection coefficient, isolation coefficient, and output power.

\section{Broadband Power amplifier design methodology}

The balanced power amplifier is a convenient method for realizing a broadband PA that has good performances especially in input/output return loss and flat gain. The balanced amplifier is constructed with two single-stage power amplifiers and two hybrid couplers as shown in Figure 1..

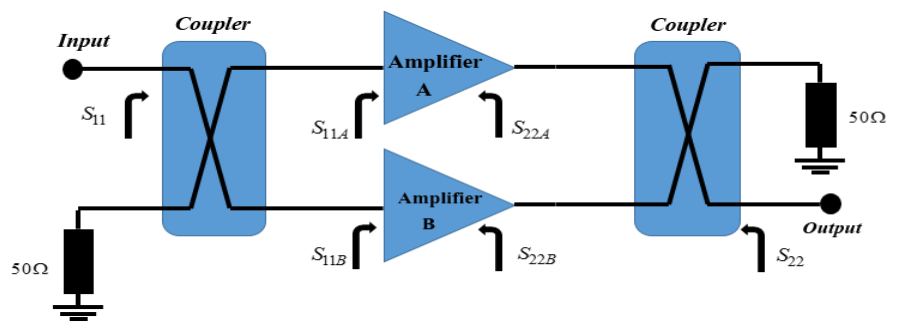

Fig. 1. Conventional topology of balanced amplifiers with hybrid couplers.

Considering the balanced amplifier architecture in Figure 1. the coupler on the left is working as the power divider and the one on the right is work as the power combiner, while the two amplifiers (Amplifier-A, Amlifier-B) are identical and have S-parameters $\left[\mathrm{S}_{-\mathrm{A}}\right]$ and $\left[\mathrm{S}_{-\mathrm{B}}\right]$ respectively. Thanks to the phase difference of the couplers, the two PA work in quadrature. it can be demonstrated that $[\mathrm{S}]$ parameters of the overall circuit (the balanced power amplifier are [11]:

$$
\begin{aligned}
& \left|S_{11}\right|=\frac{1}{2}\left|S_{11 A}-S_{11 B}\right|=0 \\
& \left|S_{12}\right|=\frac{1}{2}\left|S_{12 A}+S_{12 B}\right|=S_{12 A} \\
& \left|S_{21}\right|=\frac{1}{2}\left|S_{21 A}+S_{21 B}\right|=S_{21 A} \\
& \left|S_{22}\right|=\frac{1}{2}\left|S_{22 A}-S_{22 B}\right|=0
\end{aligned}
$$

It is clear from equations (1 - 4), that the magnitude of $S_{11}$ and $S_{22}$ will turn zero. By adopting the balanced configuration, excellent input and output matching conditions can be acquired simultaneously, while the reverse isolation and gain are kept unchanged. 
In order to design a balanced power amplifier. As a first step, a single-stage power amplifier is designed and then transformed it into a balanced amplifier configuration by incorporating the hybrid couplers.

\subsection{Design of the single-stage power amplifier}

The important step that should take into consideration in designing a power amplifier is the choice of an appropriate active device (transistor). The choice of an active device for a microwave power amplifier is very complicated. It implicates choosing an active device having an acceptable current rating with output power and power gain capability that meets the requirements of the intended application. It is also important that the chosen an active device has a breakdown voltages which will not be exceeded by the RF and DC voltages that appear through the various junctions of the transistor and that permit the gain at frequency objectives to be met by the active device.

According to the design target, we select ATF-21170 transistor [12]. ATF-21170 is a low noise enhancement-mode GaAs FET designed to be employed for low cost commercial applications in the $500 \mathrm{MHz}$ to $6 \mathrm{GHz}$ frequency range [12]. It has high linearity, low noise and high gain performance and so on, therefore, that it can satisfy our request perfectly.

The bias circuit design is very important during an amplifier design process, The aim of the bias circuit is to set the quiescent point that is the Ids and Vgs for an active device that causes it to work in the preferred region. From a general perspective, there are different kinds of biasing networks although in power amplifier applications. Low complexity is required. One commonly and probably used method is to utilize a radial stub immediately after $\lambda / 4$ high impedance bias line that will facilitate to realize proper isolation at desired $\mathrm{RF} / \mathrm{microwave}$ frequency, whatever the component to use after $\lambda / 4$ long bias line [13].

A stable and strong bias circuit to supply an appropriate quiescent operating point is important since the bias circuit will also affect the output power, gain, stability and so on. A class-A biasing point was chosen to obtain a good performance in power gain with good matching impedances and high output power. Depending on the datasheet [12]. We select the typical quiescent operating point, $\mathrm{Vds}=4 \mathrm{~V}, \mathrm{Vgs}=0.5 \mathrm{~V}$, Ids $=60 \mathrm{~mA}$.

The power amplifier may fail if the design circuit is unstable because of oscillation. There are two natures of stability namely conditional stability and unconditional stability. Conditional stability in PA presents when the stability factor $(\mathrm{k})$ less than 1 and this condition rely on the source and the load termination. It can keep the system stable for a certain range of source and load impedances [14].To contrariwise, the unconditional stability in PA happens when stability factor more than 1, ensures the circuit to be stable for all source/load impedances. For the proper running of the power amplifier, the unconditional stability must be ensured. For this, we add a taper line to the source of the transistor [15] as shown in Figure 2 .

$$
k=\frac{1-\left|S_{11}\right|^{2}-\left|S_{22}\right|^{2}+\left|S_{11} \cdot S_{22}-S_{12} S_{21}\right|^{2}}{2\left|S_{12} S_{21}\right|}
$$

Where $\mathrm{k}$ is the stability factor [14]. 
In the design of power amplifier, the matching network makes the input/output impedance transform to $50 \Omega$ impedance in the condition of good power gain and low return loss .The schematic of the proposed single-stage power amplifier is shown Figure 2; it comprises a stability network, bias network, the input and output matching networks, For machining, a "T" type matching is used at the input and the output sides of the transistor.

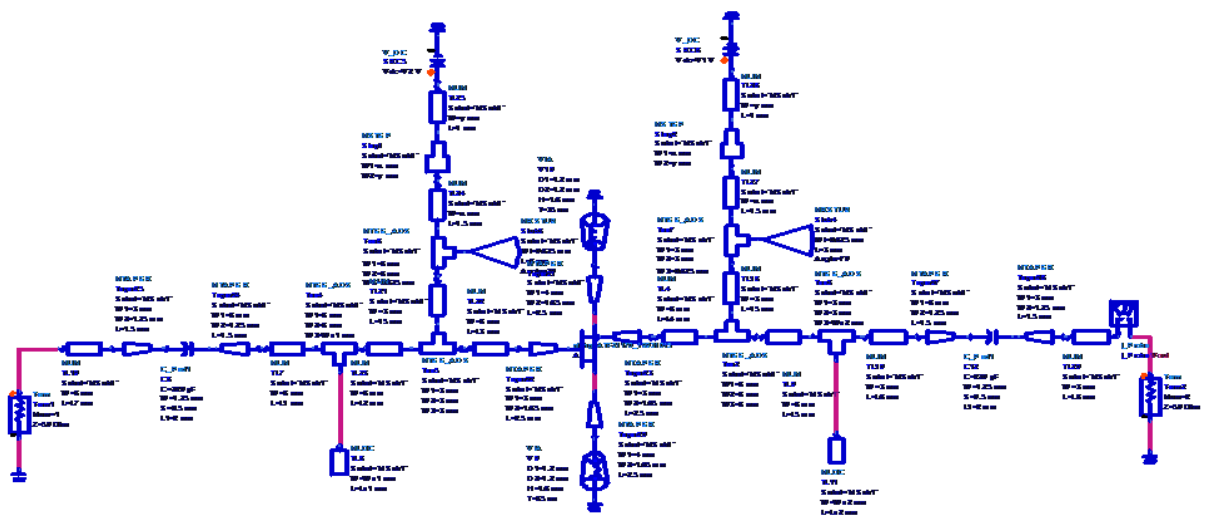

Fig. 2. The schematic of the proposed single-stage power amplifier

The simulation results of the proposed single-stage amplifier circuit are illustrated in Figures 3-5. Figure 3 presents the simulated S-parameters of the performance of the proposed single-stage power amplifier. the power gain $\left(S_{21}\right)$ of this power amplifier is about $19 \mathrm{~dB}$ with an isolation coefficient $\left(\mathrm{S}_{12}\right)$ lower than $-24 \mathrm{~dB}$ at $2.45 \mathrm{GHz}$, and the reflection coefficients are lesser than $-10 \mathrm{~dB}$ over the interested band; it can be observed that the input reflection coefficient $\left(S_{11}\right)$ is less than $-38 \mathrm{~dB}$ and the output reflection coefficient $\left(\mathrm{S}_{22}\right)$ is less than $-33.5 \mathrm{~dB}$ at $2.45 \mathrm{GHz}$. Whereas the circuit achieves a maximum saturated output power of $14 \mathrm{dBm}$ as shown in Figure 4. As depicted in Figure 5, it can be seen that $\mathrm{k}>1$ over operating frequency band. Consequently, the condition for unconditional stability of the proposed single-stage power amplifier is confirmed on the interested band, which means there is no risk of oscillations. Despite this performance but the single-stage amplifier it has a narrow band.

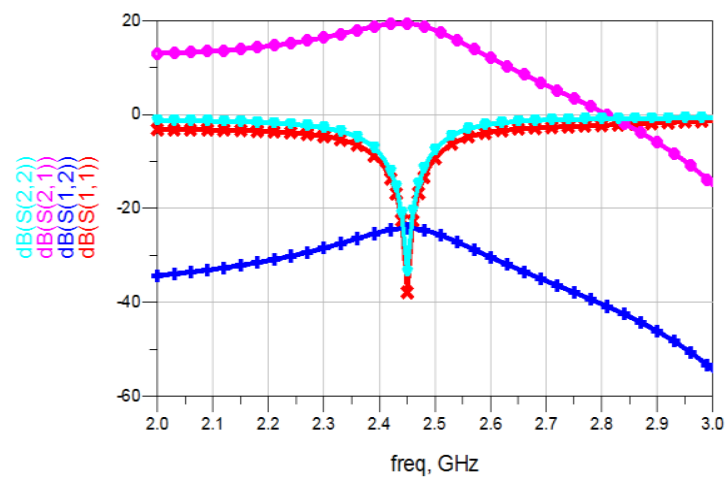

Fig. 3. S-parameters versus frequency of the proposed single-stage amplifier. 


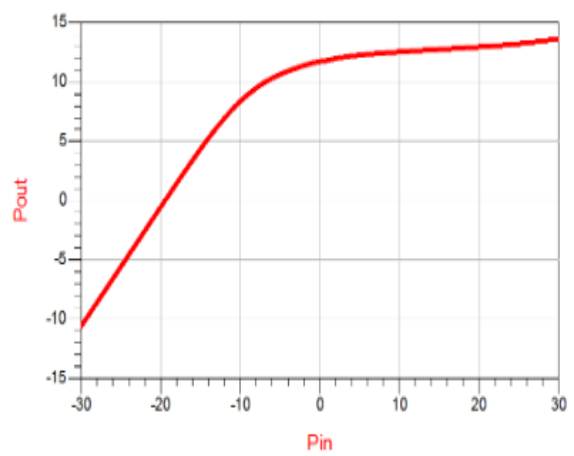

Fig. 4. Input power versus output power for $2.45 \mathrm{GHz}$.

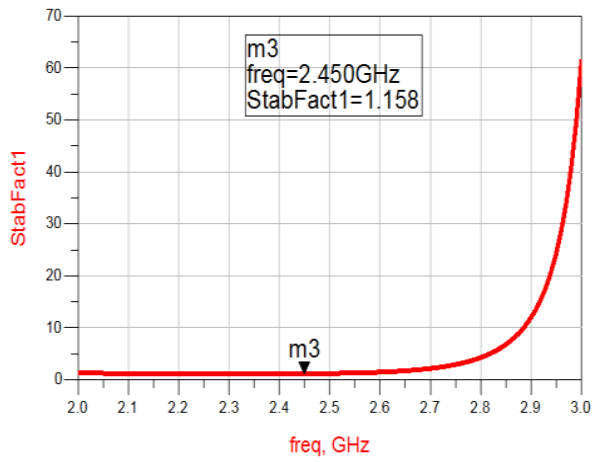

Fig. 5. Stability factor versus frequency characteristics.

\subsection{Design of the balanced power ampifier configuration}

After we have finished designing the structure of the single-stage PA we move on to the next step, which is the design of the hybrid coupler.

Due to the branch-line coupler has low-performance and large-coupler is difficult to implement, the Wilkinson power divider has been utilized in this work. The Wilkinson power divider is a type of classical one with a straightforward structure and easy to implement. The circuit has been designed on Epoxy substrate $\left(\varepsilon_{\mathrm{r}}=4.4, \tan \delta=0.025\right)$ with $1.6 \mathrm{~mm}$ thickness. The selection of the substrate should consider its permittivity and its thickness. These characteristics impact on bandwidth. Epoxy (FR4) combines good electrical features, availability and price. In order to verify the accuracy of the design, the simulation has been carried out on an Agilent ADS. The characteristic impedance is $50 \Omega$ and the center frequency is $2.45 \mathrm{GHz}$, then the length and width of the microstrip can be determined using LineCalc tool. The schematic circuit of the Wilkinson divider and simulation results are illustrated in Figure 6 and Figure 7, respectively. The results indicate that the isolation $\left(S_{32}\right)$ between port 2 and port 3 is lower than $-27 \mathrm{~dB}$. The transportation gain $\left(\mathrm{S}_{21}\right)$ is $-4.8 \mathrm{~dB}$, the reflection coefficient $\left(\mathrm{S}_{11}\right)$ of each port is also less than $-38 \mathrm{~dB}$.

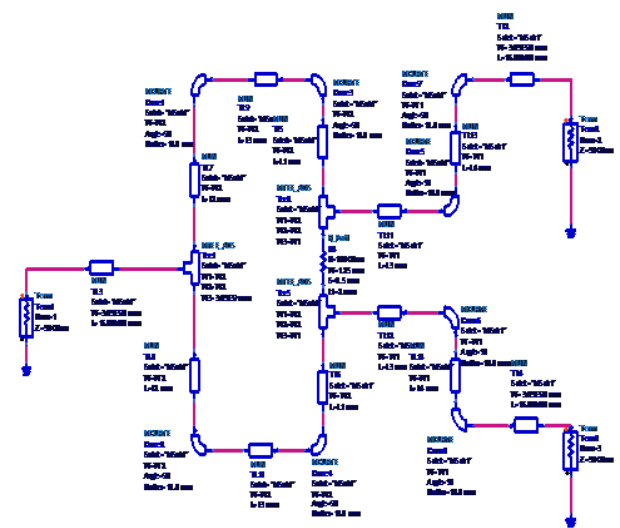

Fig. 6. The schematic circuit diagram of the Wilkinson power divider

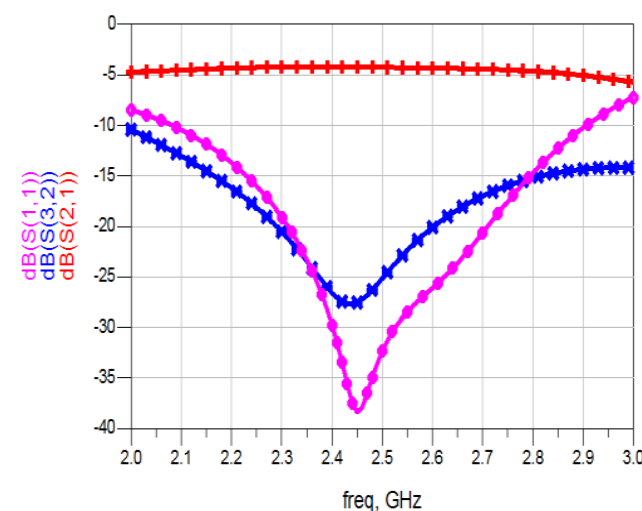

Fig. 7. The simulated results of the Wilkinson power divider 
Finally, we mix two parallel single-stage amplifiers and two power dividers. The printed circuit of the balanced power amplifier was implemented in microstrip technology using the Epoxy substrate (FR4) with a relative permittivity of 4.4 , a thickness of $1.6 \mathrm{~mm}$, a metallization thickness $\mathrm{t}=0.035 \mathrm{~mm}$ and a tangential loss of 0.025 . For more realizable results, Momentum tool is performed. Layout architecture utilized to show the location of the components and the real circuit size. The layout of the final proposed balanced power amplifier structure is presented in Figure 8, where the overall size of this balanced PA is $260 \mathrm{~mm}$ (L) * $93 \mathrm{~mm}(\mathrm{~W})$. The simulation results of the balanced power amplifier circuit are illustrated in Figures 9-11.

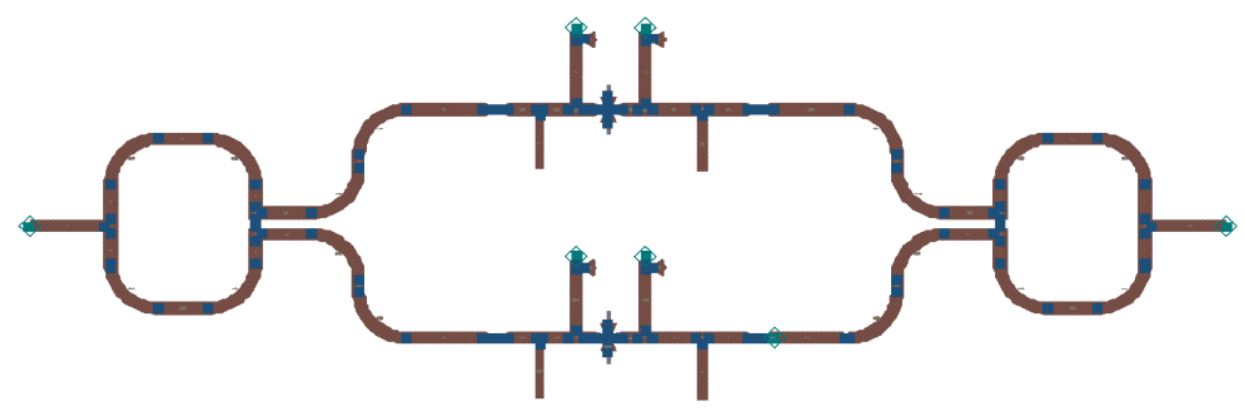

Fig. 8. The complete structure of the balanced amplifier

The S-parameter of the proposed balanced power amplifier is presented in Figure 9. For this structure, the power gain is more than $16 \mathrm{~dB}$ in the required frequency range of 2.40 $2.50 \mathrm{GHz}$ with an isolation coefficient $\left(\mathrm{S}_{12}\right)$ is less than $-28 \mathrm{~dB}$ in the operating frequency band. Note that the power gain is dropped by $3 \mathrm{~dB}$ at the peak-gain frequency for the balanced PA due to the additional loss of the Wilkinson power divider. Whereas the input and output reflection coefficient $\left(S_{11}\right.$ and $\left.S_{22}\right)$ of this amplifier are less than $-15 \mathrm{~dB}$ with the input reflection coefficient $S_{11}$ is between $-26 \mathrm{~dB}$ and $-38 \mathrm{~dB}$, while the output reflection coefficient $S_{22}$ ranges between $15 \mathrm{~dB}$ and $-19 \mathrm{~dB}$. It is clear from the result that the proposed balanced amplifier provides good matching impedances.

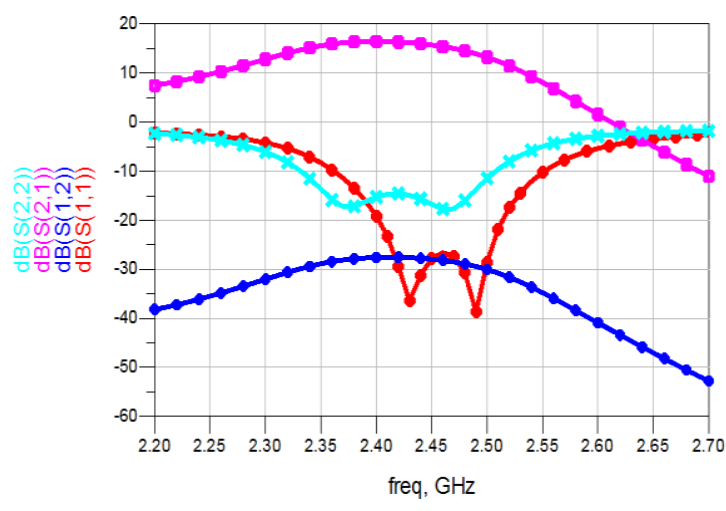

Fig. 9. S-parameters versus frequency of the proposed balanced amplifier. 
Thereby this balanced amplifier has a bandwidth equal to $100 \mathrm{MHz}$ from $2.40 \mathrm{GHz}$ to 2.50GHz. From Figures 10 and 11, we can see that the max value of the output power rise up to $16 \mathrm{dBm}$ and the stability factor of the amplifier is bigger than 2.1 that signifies the circuit is unconditionally stable.

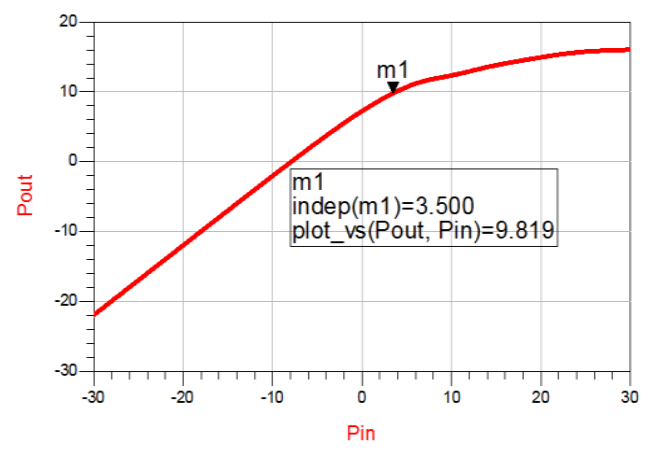

Fig. 10. Input power versus output power for $2.45 \mathrm{GHz}$

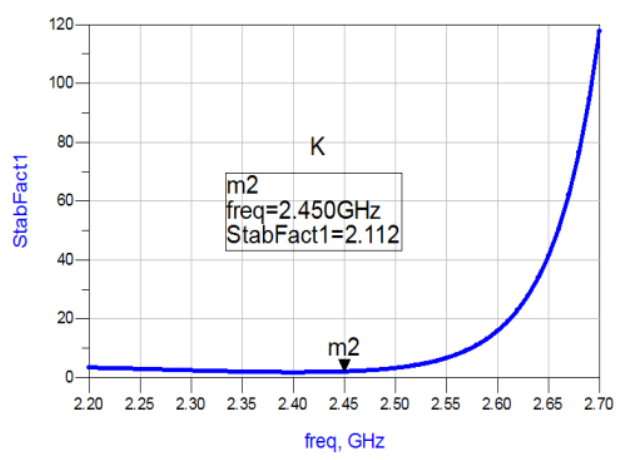

Fig. 11. Stability factor versus frequency characteristics.

After the completed the design of the balanced amplifier, a performance comparison between the proposed amplifier and other designed amplifiers in literature in terms of power gain, reflection coefficients and supply voltage are introduced in Table 1. As mentioned in Table 1, we can clearly observe that the proposed amplifier achieves a better performance compared to the cited works.

Table 1. Comparison between the proposed amplifier with published literature.

\begin{tabular}{cccccccc}
\hline Ref & Technology & $\begin{array}{c}\text { Frequency } \\
(\mathbf{G H z})\end{array}$ & $\begin{array}{c}\text { Supply } \\
\text { Voltage } \\
(\mathbf{V})\end{array}$ & $\begin{array}{c}\text { Power } \\
\text { Gain } \\
(\mathbf{d B})\end{array}$ & $\begin{array}{c}\text { Output } \\
\text { Power } \\
(\mathbf{d B m})\end{array}$ & $\begin{array}{c}\text { Input } \\
\text { Return } \\
\text { Loss } \\
(\mathbf{d B})\end{array}$ & $\begin{array}{c}\text { Output } \\
\text { Return } \\
\text { Loss }(\mathbf{d B})\end{array}$ \\
\hline$[\mathbf{1 6}]$ & GaAs FET & $1.10-3.00$ & 3 & 12 & 14.8 & -25 & -17 \\
{$[\mathbf{1 7}]$} & CMOS & $1.65-2.00$ & 10 & 10 & 18.5 & -21 & -8 \\
{$[\mathbf{1 8}]$} & Si BJT & $1.75-2.15$ & 12 & $11 \pm 0.5$ & $8 @ 1 \mathrm{~dB}$ & -22 & -18 \\
This & GaAs FET & $2.40-2.50$ & 4 & 16 & 16 & -38 & -19 \\
Work & & & & & & & \\
\hline
\end{tabular}

\section{Conclusion}

In this study, we have developed a new balanced power amplifier by using a Wilkinson power divider. This balanced amplifier was designed using the microwave simulation Advanced Design System (ADS) software. The proposed amplifier was designed by a microstrip technology and packaged GaAs FET ATF-21170. The final circuit has achieved a wide bandwidth of $100 \mathrm{MHz}$, with The $S_{11}$ and $S_{22}$ are all less than $-15 \mathrm{~dB}$, output power up to $16 \mathrm{dBm}$, and $16 \mathrm{~dB}$ power gain. Furthermore, the proposed balanced amplifier can keep more than $1 \mathrm{~dB}$ 
gain flatness at the whole operation band which shows much better performance than the conventional narrowband amplifier (single-stage amplifier).

Acknowledgments. The authors are thankful to Mr. Mohamed Latrach Professor in ESEO, engineering institute in Angers, France, for allowing us to use electromagnetic solvers available in his laboratory.

\section{References}

[1] Ng, H. T., Ziazadeh, R. M., et Allstot: A multistage amplifier technique with embedded frequency compensation. IEEE Journal of Solid-state circuits, vol. 34, no 3, p. 339-347 (1999).

[2] Gillick, M., Robertson, I. D., Et Joshi, J. S: Coplanar waveguide two-stage balanced MMIC amplifier using impedance-transforming lumped-distributed branch line couplers. IEE ProceedingsMicrowaves, Antennas and Propagation, vol. 141, no 4, p. 241-245 (1994).

[3] Akkaraekthalin, P., Jongjitaree, S., et Vivek, V: Coplanar waveguide balanced amplifier using bipolar junction transistors and backed ground-plane hybrids. In: TENCON 2001. Proceedings of IEEE Region 10 International Conference on Electrical and Electronic Technology. p. $732-735$ (2001).

[4] Nelson, B. L., Umemoto, D. K., Perry, C. B., et al: High-linearity, low DC power monolithic GaAs HBT broadband amplifiers to $11 \mathrm{GHz}$. In: Microwave and Millimeter-Wave Monolithic Circuits Symposium, Digest of Papers. p. 15-18 (1990).

[5] Seo, S., Pavlidis, D., et Moon, J. S: A wideband balanced AlGaN/GaN HEMT MMIC low noise amplifier for transceiver front-ends. In: Gallium Arsenide and Other Semiconductor Application Symposium, EGAAS 2005. European. IEEE. p. 225-228 (2005).

[6] Engelbrecht, R. S., et Kurokawa, K: A wide-band low noise L-band balanced transistor amplifier. Proceedings of the IEEE, vol. 53, no 3, p. 237-247 (1965).

[7] Imaoka, T., Banba, S., Minakawa, A., et Imai, N: Millimeter-wave wide-band amplifiers using multilayer MMIC technology. IEEE transactions on microwave theory and techniques, vol. 45, no 1 , p. 95-101 (1997).

[8] Duvanaud, C., Dietsche, S., Pataut, G., et Obregon, J: High-efficient class F GaAs FET amplifiers operating with very low bias voltages for use in mobile telephones at $1.75 \mathrm{GHz}$. IEEE Microwave and Guided Wave Letters, vol. 3, no 8, p. 268-270 (1993).

[9] Rachakh, A., El Abdellaoui, L., et Latrach, M: Design and Analysis of a New Configuration of Microwave Power Amplifier. Emerging Innovations in Microwave and Antenna Engineering, p. 364 (2018).

[10] Tajima, Y., et Miller, P. D: Design of broad-band power GaAs FET amplifiers. IEEE transactions on microwave theory and techniques, vol. 32, no 3, p. 261-267 (1984).

[11] Gonzalez, G: Microwave transistor amplifiers: analysis and design. New Jersey: Prentice hall (1997).

[12] Avago Technologies Datasheet. ATF-21170 0.5-6 GHz Low Noise Gallium Arsenide FET.

[13] Rachakh, A., El Abdellaoui, L., Zbitou, J., Errkik, A., Tajmouati, A., et Latrach, M: A Novel Design of a Microstrip Microwave Power Amplifier for DCS Application using Collector-feedback Bias. International Journal of Electrical and Computer Engineering (IJECE), vol. 8, no 3, p. 1647-1653 (2018).

[14] Kassim, S., et Malek, F: Microwave FET amplifier stability analysis using geometrically-derived stability factors. In: Intelligent and Advanced Systems (ICIAS), 2010 International Conference on. IEEE, p. 1-5 (2010).

[15] Rachakh, A., El Abdellaoui, L., Errkik, A., Tajmouati, A., et Latrach, M: A New Configuration of a Microstrip Power Amplifier Using Si-BJT in Class-A Mode for DCS Applications. In: Proceedings of the 2nd International Conference on Computing and Wireless Communication Systems. ACM. p. 58 (2017) 
[16] Ribate, M., Zbitou, J., Mandy, R., Erkik, A., et Latrach, M: Broadband GaAs FET Power Amplifier for L and S Bands Applications. The International Journal of Intelligent Engineering and Systems (IJIES), vol. 11, no 5, p. 96-104 (2018).

[17] Aniktar, H., Sjoland, H., Mikkelsen, J. H., et Larsen, T: A Class-AB 1.65 GHz-2GHz broadband CMOS medium power amplifier. In: NORCHIP Conference. 23rd. IEEE. p. 269-272 (2005).

[18] Rachakh, A., El Abdellaoui, L., Zbitou, J., Errkik, A., Tajmouati, A., et Latrach, M: A Novel Configuration of a Microstrip Microwave Wideband Power Amplifier for Wireless Application. Telkomnika, vol. 16, no 1, p. 224-231 (2018). 